\title{
From Photons to Electrons - A Correlative Workflow for Analysis and Preparation of Embedded Samples Using the LaserFIB
}

Tobias Volkenandt ${ }^{1}$, Fabián Pérez Willard ${ }^{1}$, Marcus Kaestner ${ }^{2}$, Sascha Mueller ${ }^{2}$ and Benjamin Tordoff ${ }^{1}$

${ }^{1}$ Carl Zeiss Microscopy GmbH, Oberkochen, Baden-Wurttemberg, Germany, ${ }^{2}$ Carl Zeiss SMT GmbH, Oberkochen, Baden-Wurttemberg, Germany

The complexity and functional density of electronic devices and systems is continuosly increasing, posing a challenge to material and failure analysis. It is no longer sufficient to analyze and characterize materials and structures in their isolated form. Full packages and embedded samples on the scale of millimeters to centimeters have to be investigated at a resolution of nanometers. In this contribution we present a correlative workflow from photons to electrons to solve this challenge.

Modern x-ray microscopy (XRM) offers a non-destructive way of imaging a sample in 3D at submicron resolution. A multi-resolution workflow hereby allows to start with an overview scan covering the whole sample before zooming in further for high resolution scans of chosen regions of interest. In many cases this provides valuable information about the sample, its defects and overall quality already, while keeping the sample intact. If even higher resolution is needed to complete the analysis, there is no way around electron microscopy. Using a correlative software package [1], it is possible to align the SEM surface view of the sample with the XRM dataset. Once the lower resolution overview is aligned, all subsequently imported higher resolution datasets are automatically aligned as well and displayed at their correct position in the correlative workspace. The aligned correlative data can then be used to locate buried regions of interest for SEM analysis that might not be noticeable from the sample surface alone.

However, the question remains how the SEM can gain access to the buried regions of interest. This is where the newly developed LaserFIB comes in [2]. The LaserFIB features a femtosecond (fs) laser that has been integrated with a FIB-SEM. This combination allows to perfom site-specific massive material ablation by the laser prior to high fidelity FIB polishing where needed and detailed SEM imaging. All laser processing takes place in a separate chamber attached to the instrument airlock. This avoids damage to sensitive parts in the main vacuum chamber (Fig. 1).

Figure 2 shows an application example. A small defect in one of the $\mathrm{Cu}$ microbumps has been identified by XRM. After the correlative alignment, the fs laser is used to open up a large and deep cross-section to provide SEM access to the targeted structure $860 \mu \mathrm{m}$ below the sample surface (Fig.2, on the left). The quality of the just laser-prepared cross-section is sufficient to recognize features of the sample and guide the final FIB polishing. Thus, only a small area has to be polished using the FIB, providing a smooth and artefact-free surface for high-resoultion SEM imaging of the defect (Fig. 2, on the right).

In this contribution we will introduce the system and explain all steps of the workflow in detail. Application examples will be presented, illustrating the capabilities and benefits of the LaserFIB in combination with XRM. 


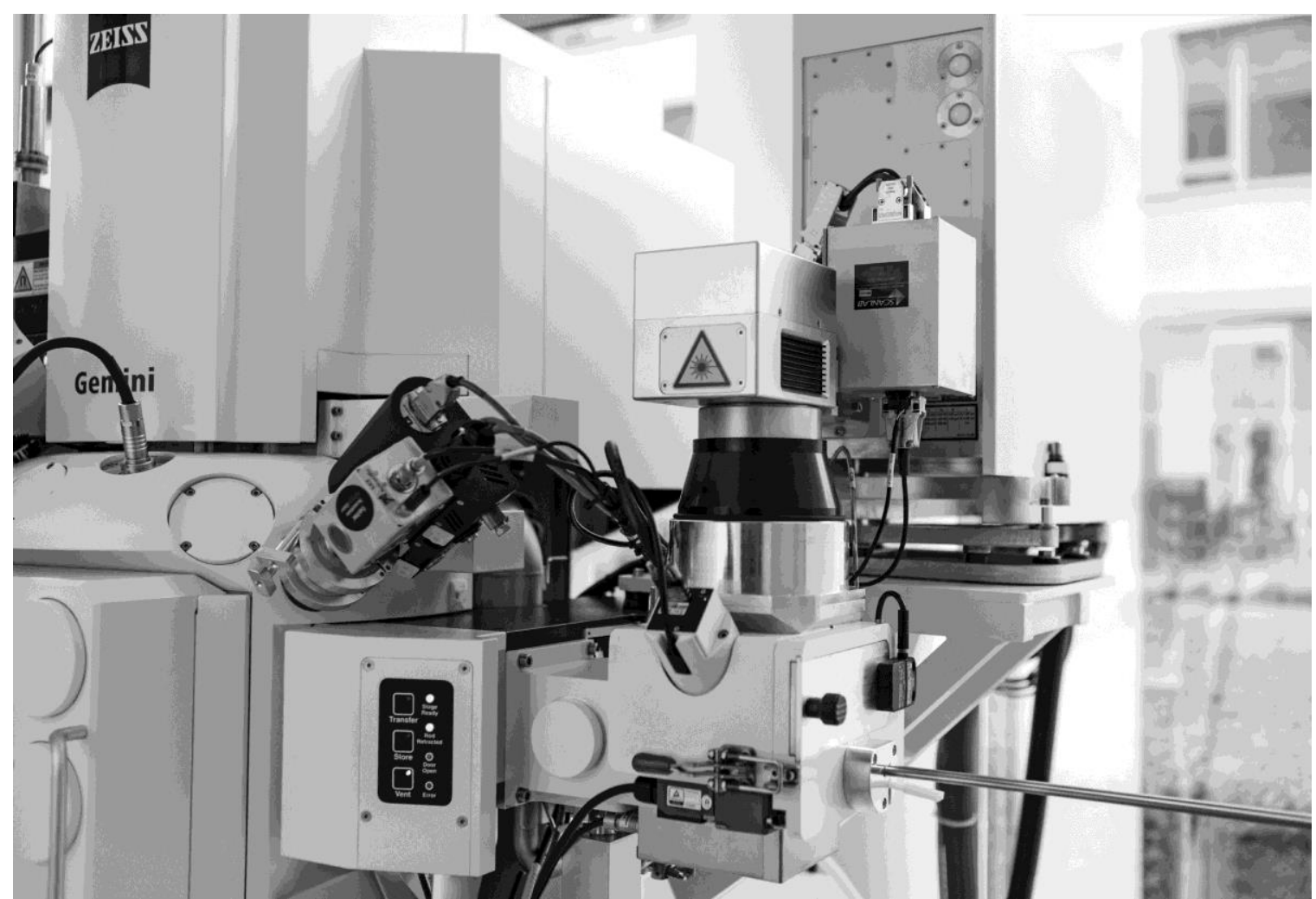

Figure 1. Picture of ZEISS Crossbeam 350 laser with fs laser attached to the airlock.

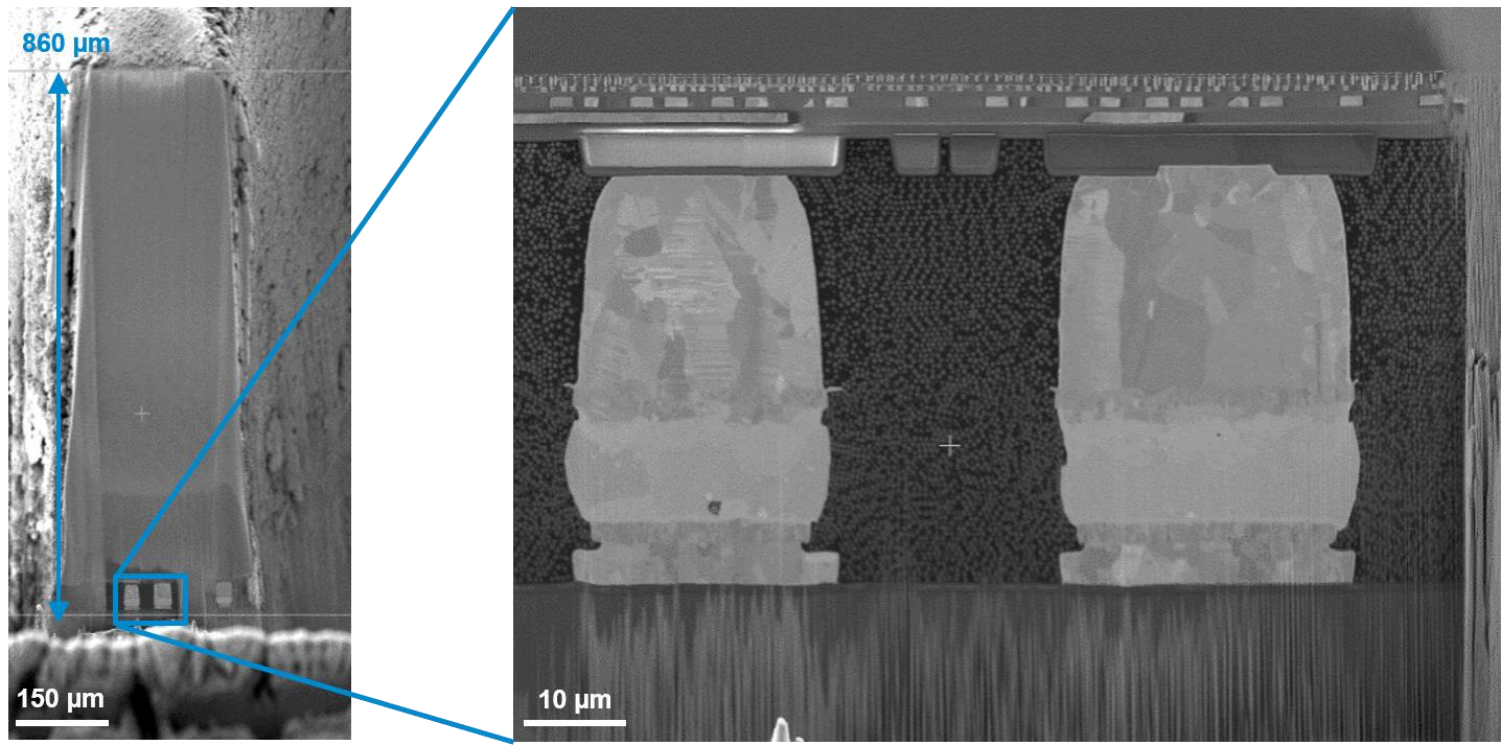

Figure 2. Overview and magnified SEM images of a defect in a $\mathrm{Cu}$ microbump $860 \mu \mathrm{m}$ below the sample surface. Access to this ROI was provided using the fs laser of the LaserFIB prior to site specific FIB polishing. In advance, the defect was located using non-destructive x-ray imaging.

\section{References}

[1] https://www.zeiss.com/microscopy/int/products/microscope-software/atlas.html

[2] https://www.zeiss.com/microscopy/int/products/fib-sem-instruments/crossbeam.html 